\title{
Geografía y problemas ambientales
}

\author{
Lizza Leal*
}

Resumen. Se abordara el tema en tres secciones: la primera ubicando la geografía en una particular clasificación de las disciplinas buscando encon trar singularidades que permitan destacar su papel como ciencia en la socie dad, proponiendo su articulaci6n como eje de las que denominaremos cien cias fácticas; en la segunda abordaremos la sociedad como una sociedad en transición de la modernidad a una nueva época que ha dado en llamarse la postmodernidad, aun como un germen, para plantear algunas preguntas y preocupaciones fundamentales que debe responder la geografía como parte de su papel en la sociedad contemporánea. La tercera y última sección, pri vilegia el papel de la geografía en las disciplinas ambientales como su más destacado papel de la sociedad en transición. Palabras clave: geografía, ciencias fácticas y formales, problemas ambientales.

Aceptado: junio 2003.

Ecòloga. Pontificia Universidad Javeriana. Estudiante Programa de Maestría en Geografía con énfasis en ordenamiento territorial. Convenio UPTC-IGAC, Bogotá. 
Se inicia esta argumentación proponiendo una premisa de análisis, que sintetizamos en una cita que nos permite definir el papel de una ciencia o disciplina en el marco social:

Las transformaciones sucesivas que ocurren en el conocimiento científico y en el contexto socioeconómico promueven un continuo cambio en los desafios y problemas enfrentados por los hombres. Buscando analizar y explicar esos problemas, a fin de proponer soluciones y prever las posibles consecuencias futuras, el conocimiento científico está siempre aceptando los desafíos y luchando para superar las cuestiones relevantes para las sociedades. Considerando las mas variadas ciencias, que son parcelas de la comunidad científica global, podemos observar que cada ciencia en particular reacciona de modo diferente a ese desafió y solicitud, y ese momento histórico puede colocarla en la posición de vanguardia o en la posición de acompañante del cortejo de las ciencias conforme a la valoración que a ella se le atribuye. (Christofoletti, Antonio. As perspectivas dos estudos geograficos).

Puede entonces decirse, siguiendo al autor, que el papel de las ciencias en la sociedad, contemporánea o no, depende básicamente de la manera como reacciona para analizar, enfrentar, prever consecuencias y proponer soluciones a los problemas centrales de la sociedad en la cual vive. De ello dependerá la importancia de su papel y el reconocimiento social.
El título de este ensayo obliga dos precisiones. De un lado a cual enfoque de geografía me refiero, pues los múltiples abordajes hacen necesario precisar o si por el contrario puedo proponer una función articuladora, una especie de pensamiento geográfico, y en el contexto especificado analizar la reacción de las vertientes de la geografía a los cambios socioeconómicos. Del otro lado igual; a cual visión de sociedad me refiero? A esta que parece predominar dominada por el paradigma capitalista, o a la sociedad inaprensible de los teóricos del cambio, o al tránsito que los teóricos ubican en la dimensión temporal de la mitad el siglo xx para hablar del fin de la modernidad y el surgimiento de la época postmoderna como un grito esperanzador que nos libere de la larga noche del tener como símbolo y medida para pasar al ser como expresión de la nueva tierra prometida?

\section{Ubicando la geografía}

En una relectura de Habermas (Vasco, Carlos Eduardo 1982). En "conocimiento e interés" los autores (Habermas, Jurgüen, 1982). proponen una nueva clasificación de las ciencias que puede sernos útil para el propósito de ubicar el papel de la geografía en la sociedad. La finalidad de intentar una nueva clasificación es evitar ciertas terminologías cargadas en una dirección y eludir el debate gastado de discusión entre ciencias del espíritu, ciencias sociales, ciencias humanas, etc. que a nuestro juicio, compartido

166 Lizza Leal 
con los autores, ha llegado a una especie de pantano conceptual que impide análisis objetivos y profundos.

Se propone allí una división entre ciencias formales y ciencias fácticas, definidas estas ultimas por su dedicación al estudio de la realidad factual e identificando tres formales -las matemáticas, la lógica y la teoría de sistemaspor su objetivo de descubrir y proponer estructuras abstractas, de tipo teórico, que modelen, reflejen o reproduzcan las regularidades encontradas en las ciencias fácticas.

A su vez las ciencias fácticas -las disciplinas prefiere el autor decir- se dividirían en disciplinas del nivel abiótico y del nivel biótico, sea que incorporen o no la vida. En ese mismo sentido las bióticas se dividirían en preantrópicas y antrópicas por razón de la incorporación o ausencia del hombre y su cultura como temática.

En la clasificación de fácticas se encuentra la geografía. En el nivel fáctico abiótico la geografía física, en el nivel fáctico antrópico las vertientes geográficas que privilegian el enfoque analítico de la relación espacio-sociedad, como la geografía humanística y en general las denominadas vertientes de la nueva geografía -marxista, idealista, alternativas- que significan la incorporación del análisis social al objeto de estudio.

He aquí una característica fundamental de la geografía que se evidencia en esta clasificación: sus diferentes abordajes ligan lo abiótico y lo biótico y a su vez privilegian alguna de las ciencias formales, principalmente las matemáticas y la teoría de sistemas. En esta característica se fundamenta la especificidad de la geografía en la división propuesta por vasco y en ella radica su importancia para servir de disciplina articuladora de las ciencias fácticas, de esa particularidad surge la posibilidad de un pensamiento geográfico como base de las disciplinas fácticas.

Se rescata en esta visión la pregunta de "Eric Wolf preocupado por las gentes sin historia" rescata la idea de que una totalidad no puede ser segmentada: si por doquier encontramos conexiones, ipor qué nos empeñamos en convertir fenómenos dinámicos e interconectados en cosas estáticas desconectadas. Luego retomare esta singularidad para proponer a la geografía como eje de las disciplinas ambientales.

La posibilidad de la geografía para analizar y proponer en la sociedad contemporánea, que permitirá la lectura de su papel, depende básicamente de su entrelazamiento con las ciencias antrópicas: Historia, economía, sociología, psicología, psicología social etc., que le permita ser disciplina de encuentro y de análisis. La geografía como ciencia tiene como objeto particular el espacio geográfico, pero solo en la explicación de lo espacial como fruto cultural puede encontrar su papel en la sociedad contemporánea. 
En mi concepto la parcelación del conocimiento actual reflejado en un gran número de disciplinas puede reducirse a los tres campos de la relectura de Habermas. La geografía aportará la visión espacial de los fenómenos y compartirá con las demás ciencias antrópicas enfoques de análisis y de propuesta. La supervivencia de las disciplinas como parcelas que reclaman para sí la totalidad no parece sostenible cuando se observa su entrelazamiento en la realidad.

Ello implica no una refundación de la geografía sino la continuidad de su evolución. Siguiendo a Ritter en "caracterización del área de la geografía”:

La geografía tradicional era marcada por la explicación objetiva y cuantitativa de la realidad que fundamentaba la escuela francesa de entonces. Fue esa escuela la que imprimió al pensamiento geográfico el mito de ciencia aséptica, no politizada, con el argumento de la neutralidad del discurso científico. Tenía como meta abordar las relaciones del hombre con la naturaleza de forma objetiva, buscando la formulación de leyes generales de interpretación. Y continúa: esa tendencia a pesar de valorizar el papel del hombre como sujeto histórico, propendía por un análisis de la relación hombre-naturaleza sin priorizar las relaciones sociales. Por ejemplo estudiaba la población, pero no la sociedad; los asentamientos humanos, pero no las relaciones sociales; las técnicas y los instrumentos de trabajo, pero no el proceso de producción. En general abstraía el hombre de su carácter social.

Fue en la posguerra cuando la realidad se volvió más compleja: el desarrollo del capitalismo y la fase monopolista, la transformación del espacio agrario con la revolución verde, la urbanización acelerada que origino las grandes metrópolis, la industrialización como signo de progreso y la universalización de las actividades guiadas por el capital y la industria. Las realidades locales no pudieron mas explicarse por sí solas. Dos grandes corrientes de pensamiento se instalaron como dicotomía: capitalismo y socialismo, con diferentes cosmovisiones que polarizaron el mundo. La geografía toma entonces dos caminos: la de ciencia que sirve al desarrollo capitalista o como una disciplina que busca no solo interpretar el mundo sino cambiarlo. Los métodos tradicionales se tornaron ineficaces pues era necesario incorporar análisis de orden económico, social, ideológico y político.

Esa tendencia de incorporación de la geografía a las ciencias antrópicas se consolida a partir de los anos 60 y principalmente en las ultimas décadas del siglo pasado, en las cuales

Una de las características fundamentales de la producción académica es la definición de abordajes que consideran las dimensiones subjetivas y por tanto singulares que los hombres en sociedad establecen con la naturaleza. Esas dimensiones son socialmente

168 Lizza Leal 
elaboradas, esto es fruto de las experiencias individuales guiadas por la cultura en la cual se encuentran insertas y resultan en diferentes percepciones del espacio geográfico y su construcción. Son esencialmente búsquedas de explicaciones más plurales que promuevan la intersección de la geografía con otros campos del saber como la antropología, la sociología, la biología y las ciencias políticas por ejemplo. Una geografía que no este apenas centrada en la descripción empírica de los paisajes, tampoco enfocada exclusivamente a la interpretación político y económica del mundo; que trabaje tanto las relaciones socio culturales del paisaje con los elementos físicos y biológicos de la cual hacen parte, investigando las múltiples interacciones entre ellos establecidas en la constitución de un espacio: El espacio geográfico. (Ritter, Carl).

Cambia entonces la noción de la geografía para ser el estudio de las relaciones entre el proceso histórico que regula la formación de las sociedades humanas y el funcionamiento de la naturaleza, a través de la lectura del espacio geográfico y del paisaje.

El espacio geográfico es entonces históricamente producido por el hombre en cuanto organiza económica y socialmente su sociedad. La percepción espacial de cada individuo o sociedad esta también marcada por lazos afectivos y referencias socioculturales. En esta perspectiva, la historicidad lo ubica como sujeto constructor del espacio geográfico. un hombre social y cultural.

\section{Ubicando la sociedad en la cual juega su papel la geografía}

En este enfoque de la geografía se buscará precisar su papel en la sociedad moderna, específicamente en el transito de la modernidad a la post- modernidad, en construcción como se ha dicho, que en vías de resumen se sintetizará en él cambio de significado de los conceptos que guiaron el accionar de la primera. Se hace referencia específicamente al cambio conceptual de las nociones de desarrollo, de progreso, de la economía y la acumulación como objetivos de la vida del hombre y de los pueblos.

Primero el desarrollo, concebido en la modernidad como un ascenso lineal medido en índices como el producto interno bruto, que cataloga la calidad de vida como la disponibilidad de lo superfluo y que pretende que todas las sociedades, sin excepción, recorran un camino trazado por aquellas que en su concepto se revelan superiores, en aplicación del concepto de verdad absoluta. Concepto unido al de progreso, medido por la presencia de la infraestructura, por el abandono de las actividades tradicionales como la agricultura para cifrar en la industria la noción de avance de los pueblos, por los avances tecnológicos y por la evolución cultural hacia un modelo que significa la asimilación a un prototipo. La globalización del camino a seguir para alcanzar el progreso y el desa- 
rrollo. Todo lo particular, toda distinta visión de vida es repudiada por apartarse del prototipo. Finalmente todo mediado por una disciplina, la economía, que dice de la mejor asignación de los recursos incluidos los humanos, de la evaluación de toda actividad por su connotación en valor.

Aclaremos que en la transición aun quedan muchos elementos de la modernidad y apenas asoman los nuevos que pugnan por su reemplazo.

La cuestión es: ¿A cuales problemas y preguntas debe responder la geografía para cumplir su papel en una sociedad contemporánea caracterizada por la transición? propongo las siguientes como una lista parcial y subjetiva:

1. La evolución y consecuencias de las relaciones globales de comercio resumidas en la denominada apertura o globalización y caracterizada hasta hoy por la formación de bloques comerciales regionales y que han cambiado el espacio económico. La geografía como ciencia por excelencia del espacio deberá responder por los cambios sociales y económicos que esa nueva configuración del espacio conlleva.

Así nos lo advierte Villanueva:

En este proceso de globalización esta apareciendo un mundo distinto, con una sociedad, una economía y una cultura diferente que minimiza y reduce la distancia espacial a tiempos y posibilidad de recepción de señales, que virtualiza la realidad y basa la economía en la información. Estos factores están haciendo surgir un nuevo mapa del mundo, con nuevas "tierras incógnitas", tierras que pasan de la "dependencia a la irrelevancia" y que lógicamente surgen de una exclusión de los flujos de riqueza y del nulo interés político y estratégico que despiertan. Son territorios fuera de control, al margen del sistema, con una estructura y funcionamiento cada vez más complejo.

Y también Dávalos en una referencia que además incita a la geografía a acometer este propósito:

La integración de la espacialidad como un agente activo en los procesos económicos y sociales esta vinculado a la transformación de las fronteras nacionales que tanto la globalización como la regionalización supranacional y subnacional han ocasionado. La perspectiva inmutable que se tenía de la nación, como una geografía establecida e incambiable estimularon sin duda el reducir a la geografía al mero escenario de los acontecimientos.

2. El fin de la época de producción Fordista, que los analistas ubican a mediados de los anos setenta, conllevó una nueva distribución espacial de la producción por una dispersión en el espacio de la misma, involucrando regiones apartadas en actividades denominadas modernas pero subalternas. Aún no hay una nueva geografía de

170 Lizza Leal 
este tipo de producción que explique el porqué de su localización y sus efectos.

3. A nivel de la economía surge una pregunta fundamental para los estudios geográficos. En el enfoque de la competitividad basada en los recursos y la ubicación, que dio origen a los postulados liberales de finales del siglo XIX, era clara la participación de la geografía en explicar la riqueza de las naciones. La nueva geografía deberá abordar ahora la espacialidad de una competitividad construida, cuyo surgimiento dio al traste con el predominio de los recursos naturales como explicación del desarrollo. Posiblemente una geografía del capital humano, del capital social y del avance de la organización y de la técnica sea la explicación al surgimiento de naciones sin mayores recursos naturales como potencias mundiales y podrá predecir cuales naciones y regiones serán los nuevos protagonistas del futuro.

4. A la preocupación por los efectos sobre el medio del modo de producción capitalista que conlleva una seria amenaza a la supervivencia de la vida y del planeta y que se expresa en el deterioro del medio físico, en la disminución de los recursos ambientales, en los cambios climáticos globales y en problemas como la ruptura de la capa de ozono, en la afectación del espacio y de las comunidades que lo habitan por fenómenos como la lluvia ácida, la disminución y contaminación de los recursos hídricos que afectan el espacio global, la contaminación de la atmósfera por la excesiva carga de $\mathrm{c} 02$ y del suelo por los desechos sólidos y líquidos que la actividad humana genera, entre otros. Esos cambios, sus efectos, las consecuencias predecibles son objeto de la preocupación de la geografía y sobre los cuales la sociedad le demanda respuestas.

5. la capacidad del medio para soportar la vida en el planeta ante una población creciente y que en el camino del desarrollo demanda mayores cantidades de energía per cápita y total para suplir las necesidades básicas y adquiridas.

6. la capacidad de autorregulación de la biosfera, que es el espacio de estudio de la geografía.

7. La cuantificación y localización de la biodiversidad que se percibe como una nueva forma de riqueza de las naciones.

8. La construcción de la geografía de la calidad ambiental y la precisión de la relación de los fenómenos locales con el universo como referencia.

9. A la nueva visión global de la economía se opone una tendencia al rescate de las culturas regionales, que encuentran en su afirmación una expresión de capital social y de apoyo ante un mundo que no cuenta sus particularidades como condicionante de su avance. Si el espacio es construcción de la cultura, la geografía deberá iden- 
tificar esas culturas y proponer organizaciones sociales y espaciales acordes con su cosmovisión.

Ello implicara un retorno a la geografía regional guiada por el concepto de región como un espacio socialmente construible.

10. Igualmente, la geografía en su papel en la sociedad contemporánea enfrentará otros retos para los cuales cuenta con el formidable arsenal de la geografía física y los avances de los sensores remotos y de la aventura aeroespacial como los satélites. Deberá responder por la información de la ocupación del espacio en actividades productivas, previendo posibles carencias de alimentos y recursos, contribuir a la bioseguridad identificando los territorios y productos que se obtienen de modos de producción que involucran riesgos a la salud, corno la utilización de plaguicidas y otros defensivos agrícolas con capacidad para causar daño a la salud y al ambiente. El tema de la bioseguridad, que surge en el espacio productivo, cobra mayor interés ante la preocupación por el terrorismo que invade al mundo.

11. Así como explico el avance de la revolución verde deberá explicar ahora el biocomercio, nacido del privilegio de la producción limpia, ecológica y orgánica.

12. Deberá agregar una nueva categoría a la descripción del paisaje que surge del regreso a la naturaleza y al privilegio de sus calidades paisajísticas, que implicará la introducción de la estética y de la armonía, que se reflejan en el auge del ecoturismo, del agroturismo, del turismo de aventura, en reemplazo del turismo de alto confort, realzado por la apreciación del ocio y del descanso como complementos de la actividad humana.

13. Finalmente una reflexión sobre la conceptualización del espacio nacida de la noción de espacio virtual que los avances en las comunicaciones y la informática hicieron posible. ¿como abordara la geografía este concepto?

Una primera respuesta nos dice que el papel de la geografía en la sociedad contemporánea pasa por responder a estas, y muchas otras preguntas, que interesan a la sociedad.

Horacio (Capel 1998:) al analizar el papel aplicado de la geografía en su artículo una geografía para el siglo xxi, refuerza lo afirmado.

Los geógrafos pueden contribuir a resolver importantes problemas del mundo actual... los problemas actuales exigen estudios y soluciones. y tanto da que sean elaborados por una u otra corporación profesional o científica. El geógrafo puede aportar soluciones a partir de su propia tradición intelectual. Pero en todo caso, lo importante es la solución ... Eso es efectivamente lo que necesitamos. Análisis penetrantes y propuestas imaginativas que permitan entender mejor la realidad actual y

172 Lizza Leal 
proponer soluciones y que hagan posible abordar con algún optimismo el futuro de la humanidad. En ese sentido, tenemos necesidad de establecer prioridades: en relación con los problemas de cada país y en relación con los problemas mundiales

De la selección de prioridades correcta dependerá en buena parte el papel de la geografía.

\section{Relacionando geografía $y$ sociedad}

De mi parte considero que el principal papel de la geografía en la sociedad contemporánea radicará en articularse como eje de las disciplinas ambientales, sin desconocer la importancia de otras intersecciones.

Se proponen dos hipótesis:

1. Es la preocupación de la sociedad por el medio ambiente, lo que hace posible intentar leer la importancia de la geografía en la sociedad Contemporánea por ser elemento central de la explicación medioambiental.

2. Presentar a la geografía y a las disciplinas ambientales como dos ramas del conocimiento que se encuentran porque evolucionan paralelamente en el tiempo, en el paso de lo territorial a lo espacial, en la incorporación de conocimientos de otras disciplinas para intentar desde sus abordajes explicaciones del mundo, en su paso de ser ciencias básicamente naturales descrip- tivas (geografía física y ecología) a ser ciencias sociales analíticas, o Fácticas antrópicas como las he denominado. Todo ello como explicación de la creciente importancia de ambas disciplinas, que solo puede ser posible si abordan visiones integrales, o brindan sustento para ello a otras ramas del conocimiento.

En efecto, la nueva geografía y el interés por la ecología, que luego devino en las disciplinas ambientales, nacen a mediados del siglo pasado como fruto del desencanto con los efectos de las visiones dominantes de desarrollo y progreso, insatisfecha la geografía porque sus instrumentos y métodos no lograban explicar la complejidad de la sociedad de entonces e insatisfecha la ecología porque el modo de producción dominante no incorporaba sus preocupaciones por el deterioro de los ecosistemas.

Ambas irrumpieron en el universo de las disciplinas bióticas antrópicas, la geografía aportando la espacialidad de los fenómenos, la ecología describiendo sus efectos sobre el medio natural. Las dos bajo una misma preocupación y objeto: las relaciones del medio físico, el biotopo ampliamente descrito y analizado por la geografía, y la biocenosis o las comunidades cuya vida y relación el medio soporta.

Es difícil diferenciar el objeto de estudio de las dos disciplinas, aunque la geografía por su antigüedad debe ser considerada como la primera y más 
importante de las disciplinas ambientales y reconociendo que de la descripción de los cambios en el espacio surgió la preocupación ecológica.

Montañez 1997 nos brinda algunas razones que ligan geografía y medio ambiente.

1. geografía y medio ambiente se unen por diversas razones relacionadas con dos características centrales del pensamiento científico, social y político del final del segundo milenio, en el cual el espacio parece transformarse en el modo privilegiado de pensar y actuar al fin de siglo, al punto que muchos pensadores coinciden en identificar esta tendencia como una de las señales del surgimiento del pensamiento posmoderno.

2. De esta manera la geografía se ve renovada ya que su énfasis en la dimensión espacial como objeto de estudio y su carácter de disciplina social ambiental, corresponden a las dos grandes preocupaciones de la humanidad al finalizar el siglo $\mathrm{XX}$.

3. El movimiento ambientalista surge y se alimenta de las limitaciones y efectos negativos de la implementación del proyecto modernizador sobre la superficie de la tierra. Es en este marco que la geografía, entendida como disciplina cuyo objetivo busca describir y explicar la dimensión espacial de las relaciones entre naturaleza y cultura, se ha visto fortalecida por el cú- mulo de interrogantes que con frecuencia se le formulan en el contexto actual.

4. La geografía como disciplina científica que desde su propio origen tuvo como objetivo el examen de las relaciones entre la sociedad y el medio en su dimensión espacial, puede contribuir de manera significativa al avance del conocimiento ambiental en general y del manejo de ambientes específicos en territorios particulares.

Finalmente que gana la geografía al transformarse en el eje de las disciplinas medioambientales? ¿Queda inmersa en las ciencias sociales? ¿ pierde su contenido especifico?

Aquí se liga el discurso de Habermas en la visión de Vasco que incluí buscando primero ubicar la geografía y ahora para enmarcarla en las ciencias sociales que en esa clasificación se incluyen en las fácticas antrópicas. Afirma Habermas que hay tres estilos de trabajo en las ciencias sociales que están caracterizadas por tres pares de verbos:

El primer par se compone de "predecir y controlar" que desarrolla la geografía física, el segundo por los verbos "ubicar y orientar" que desarroló la geografía humanística y sus afines y el tercero por "develar y liberar" que la geografía desarrollaría en la unión de las disciplinas ambientales al dejar al descubierto los efectos de la opresión de la sociedad y del ambien- 
te por el paradigma de la modernidad y contribuir al arribo de una nueva época.

Concluyendo: creo que la geografía desarrollara su papel en la sociedad contemporánea convirtiéndose en el eje articulador de las ciencias fácticas a través de la construcción de un pensamiento geográfico que permita reunir los compartimentos del conocimiento en un discurso de encuentro y de síntesis, que solo ella puede cumplir ese papel porque en sus abordajes liga lo biótico y lo abiótico, que ese papel de eje articulador comienza con las ciencias ambientales, por su objeto común de estudio, por la necesidad de un abordaje espacial, porque como ciencia se justifica en su respuesta a las inquietudes de una sociedad en transición en la cual lo ambiental es una preocupación central y, finalmente, porque en ese papel amplia a la totalidad del campo de las ciencias sociales sus métodos de trabajo. 


\section{Bibliografía}

CAPEL Horacio. Una geografía para el siglo XXI. Universidad de Barcelona. Espana. 1998.

CHRISTOFOLETTI, Antonio. As perspectivas dos estudos geograficos.

Internet: < URL: http:// www.sites.oul.com.br/ivairr

DAVALOS, Elisa. El proyecto moderno del saber científico y la postmodernidad. Revista de la facultad de economía. Año vii, $\mathrm{N}^{\circ} 21$.

HABERMAS, Jurguen. Conocimiento e interés. Traducido por Guillermo Hoyos V. Ideas y valores, $\mathrm{N}^{\circ}$. 42-45.1973-1975, pp. 6-76.

MONTANEZ, Gustavo. Geografía y medio ambiente, enfoques y perspectivas. Ediciones Universidad de la sabana. 1997.

RITTER Carl. Caracterización del .área de la geografía. Internet: < URL:

http:// www.sites.oul.com.br/ivairr

VASCO Carlos E. Tres estilos de trabajo en las ciencias sociales. Documentos ocasionales CINEP. 1990.

VILLANUEVA, José. Algunos rasgos de la geografía actual. Revista bibliográfica de geografía y ciencias sociales. universidad de Barcelona Vol. VII, $\mathrm{N}^{\circ} 342,15$ de enero de 2002 .

WOLF, Eric. Europa y la gente sin historia. México, FCE.

176 Lizza Leal 


\section{Indicaciones para presentación de artículos para la revista Perspectiva Geográfica}

\section{Formato de presentación}

- Word

- Papel blanco bond, tamaño carta

- Márgenes: Izquierda, superior y derecha: $3 \mathrm{~cm}$. Inferior: $2 \mathrm{~cm}$.

\section{Texto}

- Digite el texto a espacio sencillo, utilice Times New Roman, tamaño 12.

- Título del trabajo: centrado, negrilla, mayúsculas.

- A doble espacio: nombre del autor en minúsculas, negrilla con asterisco como llamado para anotar: profesión, títulos e institución donde trabaja.

- Resumen: escriba la síntesis del trabajo en no más de 100 palabras.

- Palabras clave: identifique las palabras principales del trabajo.

- El resumen y las palabras clave, deben venir en español, inglés y francés.

- Títulos del trabajo, numeración arábiga centrados, mayúsculas fijas.

- Subtítulos: izquierdo, negrilla y minúsculas.

- Siguientes subtítulos: izquierdo, minúsculas para los siguientes aportes utilice literales.

\section{Tablas y figuras}

- Enviarlas en archivos separados especificando el Software, en tamaño carta con las márgenes ya mencionadas.

- Las tablas y figuras deben ser enumeradas correlativamente.

- Las figuras (gráficos, mapas, etc.) deben ser presentados en original, tamaño carta.

\section{Referencias bibliográficas}

- Citas textuales cortas incluidas en el texto principal van entre comillas, con su respectiva referencia (apellidos del autor, año de publicación y página exacta de donde se extrajo la cita).

- Transcriba las citas textuales demasiado largas con indentación izquierda a renglón sencillo, sin comillas y con referencia bibliográfica exacta.

- Las referencias generales a una fuente, identificarlas con el apellido del autor y el año de publicación.

- La lista de referencias bibliográficas deben aparecer alfabéticamente, utilice normas ICONTEC para su presentación. 


\section{Maestría en Geografía Énfasis en Ordenamiento Territorial}

Dirigido a: Geógrafos, licenciados, ingenieros y otros profesionales de la ciencias sociales y naturales que demuestren genuino interés por la investigación geográfica aplicada al ordenamiento territorial.

Modalidad: presencial en horario de 7:00 a 11:00 de la mañana.

Ingreso: anual. Inscripciones: septiembre a noviembre.

Título: Magister en Geografía, énfasis en ordenamiento territorial.

\section{Diplomados}

\section{- EN ORDENAMIENTO TERRITORIAL}

Dirigido a: Funcionarios públicos, consultores y profesionales de las ciencias naturales y sociales, relacionados con la dirección, ejecución, supervisión y/o asesoría de planes de ordenamiento territorial, o interesados en adquirir conocimientos y desarrollar habilidades en el manejo de metodologías aplicables a procesos de OT.

- INSTRUMENTOS DE GESTIÓN URBANA PARA EL ORDENAMIENTO TERRITORIAL.

Dirigido a: Alcaldes, funcionarios públicos, consultores, planificadores, geógrafos, urbanistas, arquitectos e interesados en la temática del Ordenamiento Territorial.

- GEOGRAFIA DE LA SALUD

Dirigido a: Profesionales de la medicina, enfermería, administración hospitalaria, geografía y todos aquellos interesados en conocer la perspectiva geográfica de la medicina y salud. 


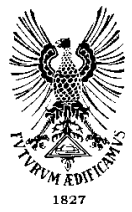

CONVENIO UNIVERSIDAD PEDAGÓGICA Y

TECNOLÓGICA DE COLOMBIA

INSTITUTO GEOGRÁFICO AGUSTÍN CODAZZI

PROGRAMA DE ESTUDIOS DE POSGRADO EN GEOGRAFÍA -EPG-

Código ICFES: 11066910000150011110

Renovación autorización de funcionamiento:

Resolución 1455 de junio 27-2002, MEN

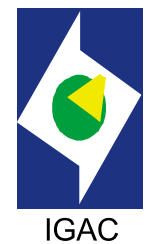

\section{Estudiantes EPG Decimoquinta Cohorte (2003 - 2004)}

GLORIA STELLA RAMÍREZ OSPITIA
Ingeniera Agrónoma
Universidad Central de Venezuela, Caracas
MARIA FERNANDA GALVIS LAGOS
Ingeniera Catastral y Geodesta
Universidad Distrital Francisco José de
Caldas, Bogotá
ANA PATRICIA GOMEZ JARAMILLO
Arquitecta
Architectural Association, Londres
ORLANDO ALBERTO TOLEDO LUGO
Ingeniero Forestal
Universidad del Tolima, Ibagué
LUISA CENOBIA LASTRE ZOLA
Licenciada
Universidad de Córdoba, Montería
ALBA NATALIA FLOREZ ZAMBRANO
Bióloga
Universidad Nacional de Colombia, Bogotá
ANDRES ALIRIO PORTILLA MONTENEGRO
Universidad de Nariño, Pasto

GLORIA STELLA RAMÍREZ OSPITIA

Ingeniera Agrónoma

LIZZA MELINA LEAL CALDERON

Ecóloga

Pontificia Universidad Javeriana, Bogotá

DAVID CONDORI GUTIERREZ

Ingeniero Agrónomo

Universidad Mayor de San Simón, Cochabamba, Bolivia

JORGE LUIS CEBALLOS LIEVANO

Ingeniero Geógrafo

Fundación Universitaria Jorge Tadeo

Lozano, Bogotá
CRISTIAN CLAUDIO JUAN BOBADILLA BUSTOS
Ingeniero Forestal
Pontificia Universidad Católica de Chile, Santiago

\section{CARLOS EDUARDO MARTINEZ \\ DELGADILLO \\ Ingeniero Ambiental \\ Fundación Universidad Central, Bogotá}

JOSE ARMANDO SANTIAGO GARNICA

Geógrafo

Universidad de los Andes, Mérida, Venezuela 
